\title{
Circulating adipokines in children with nonalcoholic fatty liver disease: possible noninvasive diagnostic markers
}

\section{Amal Ahmed Mohameda, Said Sabryb, Asmaa Mahmoud Abdallahc, Naglaa Adly Abd Elazeem ${ }^{\text {, }}$ Doaa Refaey', Hebat Allah Fadel Algebaly', Gamal Abo El Fath' ${ }^{\mathrm{g}}$, Heba Omar ${ }^{\mathrm{h}}$}

National Hepatology and Tropical Medicine Institute, Egypt; Damanhur National Medical Institute, Egypt; King Abdul-Aziz University, Jeddah, Kingdom of Saudi Arabia; Beni Suef University; Benha University, Egypt; Cairo University; Ain Shams University, Egypt

\section{Abstract}

${ }^{a}$ Department of Biochemistry, National Hepatology and Tropical Medicine Institute, Egypt (Amal Ahmed Mohmmed); ${ }^{\mathrm{b}}$ Clinical Pathology, Damanhur National Medical Institute, Egypt (Said Sabry); 'Department of Clinical Nutrition, Faculty of Applied Medical Science, King Abdul-Aziz University, Jeddah, Kingdom of Saudi Arabia (Asmaa Mahmoud Abdallah); ${ }^{\mathrm{D}}$ Medical Biochemistry Department, Faculty of Medicine, Beni Suef

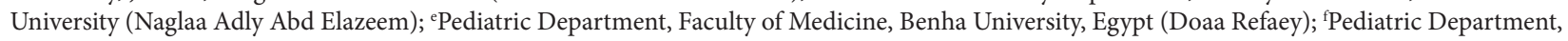

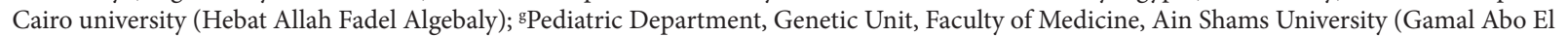
Fath); ${ }^{\text {E}}$ ndemic Medicine and Hepatology Department, Faculty of Medicine, Cairo University (Heba Omar), Egypt

Conflict of Interest: None

Correspondence to: Heba Omar, MD, GCSRT, Endemic Medicine and Hepatology Department, Faculty of Medicine, Cairo University, Egypt, e-mail: hebaomar1202@hotmail.com

Ethical approval: This study was approved by the ethics committee of Faculty of Medicine, Banha University

Received 18 December 2016; accepted 27 March 2017; published online 25 April 2017

DOI: https://doi.org/10.20524/aog.2017.0148

\begin{abstract}
Methods A prospective case-control study was conducted, which included 101 obese children with biopsy-proven NAFLD and 57 age- and sex-matched controls. The overall mean age of (10.08 \pm 3.12 years. All underwent a full clinical assessment, routine laboratory calculated and circulating chemerin and adiponectin were evaluated using ELISA.

Results Elevated serum chemerin and decreased serum adiponectin were significantly associated (inelihood of exhibiting NAFLD. Receiver operator characteristic curve chemerin, at a cutoff value of $186.7 \mathrm{ng} / \mathrm{mL}$, yielded a sensitivity and specificity of $56.44 \%$ and $87.72 \%$ respectively $(\mathrm{P}<0.001)$, whereas adiponectin, at a cutoff value of $2.4 \mu \mathrm{g} / \mathrm{mL}$, had a sensitivity and specificity of $74.26 \%$ and $3.51 \%$ respectively $(\mathrm{P}<0.001)$. Furthermore, body mass index, aspartate transaminase, alanine transaminase, triglycerides, and gamma-glutamyl transferase adiponectin $(\mathrm{P} \leq 0.001)$.
\end{abstract}

Conclusion Circulating chemerin and adiponectin could serve as simple noninvasive diagnostic Keywords Diagnosis, noninvasive, nonalcoholic fatty liver disease, obese children

Ann Gastroenterol 2017; 30 (4): 1-7 


\section{Introduction}

Nonalcoholic fatty liver disease (NAFLD) has become the most common cause of chronic hepatitis and liver-related morbidity and mortality worldwide. The histopathological features of NAFLD include steatohepatitis and fibrosis, with an increased risk of progression to end-stage liver disease, hepatocellular carcinoma (HCC), and indication for liver transplantation $[1,2]$.

Childhood obesity is currently considered a global pandemic and represents one of the most serious public health problems of the $21^{\text {st }}$ century [3]. The estimated prevalence of NAFLD in the child population is about $10 \%$, reaching 40-70\% among obese children [4]. Frequently associated with a low-grade systemic inflammatory status, obesity is the most common risk factor for pediatric NAFLD [5]. A low-grade systemic inflammatory status influences lipid metabolism, leading to changes in adipokine production by adipose tissue and subsequent interference with normal insulin function and development of insulin resistance [6,7], which represents the first "hit" in the NAFLD pathogenesis. The subsequent release of proinflammatory cytokines could cause a second hit, leading to nonalcoholic steatohepatitis (NASH) [8].

Early diagnosis and proper management of NAFLD may reduce its liver-related morbidities [9]. For the moment, histopathological evaluation of liver biopsy samples remains the gold standard for accurate diagnosis of NAFLD. However, being invasive, costly, and carrying a possible risk of sampling errors and serious complications [10], it would be desirable if biopsy could be replaced by noninvasive and affordable biomarkers for a precise diagnosis of NAFLD.

Serum concentrations of classic adipokines (e.g. adiponectin) are altered in NAFLD patients. Recently, the role of novel adipokines (e.g. chemerin) in the NAFLD pathogenesis has emerged. However, studies on serum concentrations of such adipokines in obese children with NAFLD are lacking [4].

The current study aimed to evaluate circulating adipokines (chemerin and adiponectin) as noninvasive biomarkers for NAFLD diagnosis in obese non-diabetic children and to explore their possible correlation with histopathological features of the disease.

\section{Patients and methods}

\section{Research design and study population}

This was a case-control study using prospectively collected data from 101 biopsy-proven NAFLD children below the age of 18 , as well as 57 age- and gender-matched healthy volunteers, consecutively recruited from the outpatient clinic of pediatric department, Faculty of Medicine, Banha University, during the period from January to June 2016. Patients with chronic viral hepatitis due to virus $\mathrm{C}$ or $\mathrm{B}$, chronic alcohol intake, autoimmune hepatitis, Wilson's disease, $\alpha 1$ antitrypsin deficiency, significant endocrinal disease, drug toxicity and those on total parenteral nutrition were excluded. Additionally, diabetic children and those taking drugs affecting lipid or carbohydrate metabolism were not allowed to enroll.

The study was conducted according to the guidelines laid down in the Declaration of Helsinki 1975, after receiving approval from the clinical research ethics committee of Faculty of Medicine, Banha University. Written subject assent and parental consent were obtained for all patients prior to their enrollment into the study.

\section{Outcomes}

The primary outcomes of the current study were as follows: establishing the usefulness of serum chemerin and serum adiponectin as noninvasive diagnostic biomarkers for NAFLD in obese non-diabetic children, setting a cutoff value of those markers at which NAFLD diagnosis could be highly suspected, and exploring the potential correlation between serum concentrations of candidate biomarkers and histopathological changes of NAFLD.

\section{Participants' assessment}

Children were defined as obese if their body mass index (BMI) was equal to or above the $95^{\text {th }}$ percentile [11]. Patients suspected to have NAFLD on the basis of clinically evident hepatomegaly, elevated hepatic necro-inflammatory markers (aspartate transaminase [AST] and alanine transaminase [ALT]), or fatty liver changes on abdominal ultrasound, had their diagnosis of NAFLD confirmed by liver biopsy. An ultrasound-guided liver biopsy was performed by a single expert hepatologist in all patients, after they had provided informed consent and when platelet count and coagulation profile were within an acceptable range, using an automatic true-cut (18G) needle. To be satisfactory, a specimen had to be at least $10 \mathrm{~mm}$ in length and contain at least 5 complete portal tracts. Biopsy specimens were stained with hematoxylin-eosin and silver.

The histopathological interpretation of the liver biopsy was determined according to the NAFLD activity score (NAS) [12], which includes individual scores for grades of hepatic steatosis, lobular inflammation, and hepatocellular ballooning, as well as the staging of hepatic fibrosis. The fibrosis score ranges from 0 (absence of fibrosis) to 4 (probable or definite cirrhosis). In particular, stage 1 is further subdivided into stage 1a (delicate perisinusoidal zone 3 fibrosis), $1 \mathrm{~b}$ (dense perisinusoidal zone 3 fibrosis), and 1c (portal fibrosis only), the latter referring to the pattern of fibrosis sometimes seen in severely obese and in pediatric NAFLD patients.

NAS score ranges from 0 to 8 , with a score of 1 or 2 corresponding to definitely not NASH, while a NAS score 5-8 is suggestive of definite NASH. Scores of 3 and 4 are considered borderline for NASH.

All participants underwent a full clinical examination, abdominal ultrasound, BMI measurement (weight in 
$\mathrm{kg} /$ height in $\mathrm{m}^{2}$ ), routine laboratory investigations (AST, ALT, alkaline phosphatase, $\gamma$-glutamyl transferase [GGT], albumin, and bilirubin) using conventional automated analyzers in the biochemistry laboratory at NTMR reference lab. All biochemical analyses were performed in a "blinded" manner.

\section{Blood samples and biomarker qualification}

Venous blood samples were collected from all study participants after an overnight 12 -h fast to measure fasting blood glucose, fasting insulin, total cholesterol, and triglycerides. Insulin resistance was determined by the homeostatic model assessment of insulin resistance (HOMA-IR) equation [13]:

Fasting insulin $(\mu \mathrm{U} / \mathrm{mL}) \times$ Fasting glucose $(\mathrm{mg} / \mathrm{dL}) / 405$

Fasting serum chemerin was measured using commercial ELISA kits (BioVender). The intra- and inter-assay coefficients of variation of chemerin ranged from $5.1 \%$ to $7.0 \%$ and $6.9 \%$ to $8.3 \%$ respectively. All assays were conducted according to the manufacturer's instructions.

Fasting serum adiponectin levels were measured in duplicate using an enzyme-linked immunosorbent assay (ELISA) kit (Quintikine) obtained from R\&D Systems (Wiesbaden-Nordenstadt, Germany). Each serum sample was diluted 100-fold.

\section{Statistical analysis}

Data were entered, validated, and analyzed using STATA 14 software. Patients' demographic and routine laboratory data were expressed as number (percent) for categorical variables and as mean ( \pm standard deviation [SD]) for continuous variables. Continuous variables were tested for normality using histograms and sktest. Those variables with a P-value $<0.05$ on Skewness-Kurtosis test were considered not to be normally distributed. The Spearman correlation coefficient $(r)$ was used for binary correlations. Comparisons between cases with NAFLD and controls were made using Student's $t$-test or the Mann-Whitney test, as appropriate. The chi-square test was used for comparisons of categorical data. Associations between clinical characteristics, laboratory markers, and NAFLD diagnosis were investigated using univariate and multivariate logistic regression models. Data were reported as odds ratios (OR) with $95 \%$ confidence intervals (95\%CI). The diagnostic performance of chemerin and adiponectin in the prediction of NAFLD was evaluated using receiver operator characteristic (ROC) curves. The area under the ROC curve, sensitivity, specificity, positive predictive value, negative predictive value and the 95\%CI were used as indexes of accuracy. All statistical analyses were based on two-sided hypothesis tests with a significance level of $\mathrm{P}<0.05$.

\section{Results}

\section{Characteristics of the study population}

One hundred one children with biopsy-proven NAFLD were evaluated in the current study, as well as 57 normal age- and sex-matched controls. Circulating chemerin levels were significantly higher in NAFLD children compared with normal controls $(205.69 \pm 53.22$ vs. $153.19 \pm 42.72 \mathrm{ng} / \mathrm{mL}$; $\mathrm{P}<0.001)$, whereas serum adiponectin levels were significantly lower in the NAFLD group $(3.01 \pm 2.07$ vs. $10.63 \pm 3.13 \mu \mathrm{g} / \mathrm{mL}$; $\mathrm{P}<0.001)$. Comparisons of clinico-demographic and laboratory data between NAFLD patients and controls are presented in Table 1. As expected, significantly higher BMI, AST, ALT, alkaline phosphatase, GGT, triglycerides, glucose, and fasting insulin, indicative of a high metabolic burden, were observed in NAFLD children compared to controls.

\section{Comparisons within different specific histopathological lesions}

Overall, the mean \pm SD NAS score was $5.73 \pm 1.04$ in the NAFLD obese children group. The percentage of patients with a NAS score of 5 or more (those with steatohepatitis) was $69.30 \%$. The percentage of patients with a steatosis grade of less than $33 \%$ was $73.27 \%$, while $26.73 \%$ had a steatosis grade of more than $33 \%$ (Fig. 1). The incidences of lobular inflammation with fewer than 4 foci and more than 4 foci per high power field (HPF) were $48.51 \%$ and $51.49 \%$ respectively (Fig. 1). The most common fibrosis grade in our NAFLD patients was F1 (80\%) (Fig. 1). When circulating chemerin and adiponectin were compared across groups with specific histological changes in NAFLD patients $(n=101)$, no statistically significant difference was observed in chemerin levels between groups of low vs. high steatosis, lobular

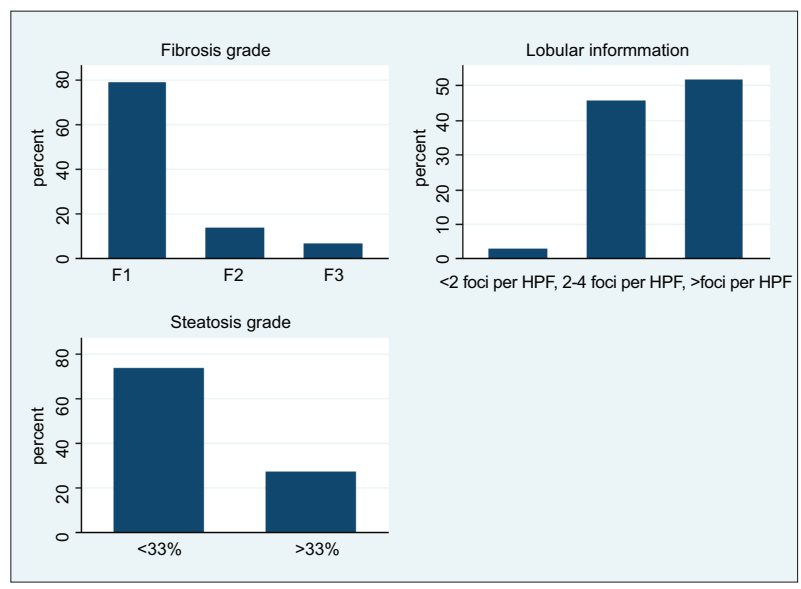

Figure 1 The frequency (percentage) of histologic findings in the nonalcoholic fatty liver disease group. The degree of steatosis (top), lobular inflammation degree based on foci of lobular inflammation in high power field of microscopic view (middle), and fibrosis degree (bottom) 
Table 1 Demographic and laboratory characteristics of the study participants $(n=158)$

\begin{tabular}{|c|c|c|c|}
\hline Variable & $\begin{array}{l}\text { Control } \\
\mathrm{N}=57\end{array}$ & $\begin{array}{c}\text { NAFLD } \\
\mathrm{N}=101\end{array}$ & P-value \\
\hline Age (yrs) & $9.53 \pm 3.08$ & $10.40 \pm 3.11$ & 0.09 \\
\hline $\begin{array}{l}\text { Sex, n (\%) } \\
\text { Female } \\
\text { Male }\end{array}$ & $\begin{array}{l}37(64.91) \\
20(35.09)\end{array}$ & $\begin{array}{l}72(71.29) \\
29(28.71)\end{array}$ & 0.405 \\
\hline BMI $\left(\mathrm{kg} / \mathrm{m}^{2}\right)$ & $17.81 \pm 3.19$ & $31.02 \pm 3.92$ & $<0.001$ \\
\hline AST (U/L) (range 0-40) & $36.82 \pm 16.65$ & $61.20 \pm 25.58$ & $<0.001$ \\
\hline ALT (U/L) (range 0-40) & $36.60 \pm 27.99$ & $68.48 \pm 23.85$ & $<0.001$ \\
\hline Alkaline phosphatase (U/L) (range 30-129) & $195.70 \pm 42.50$ & $245.61 \pm 103.15$ & 0.0006 \\
\hline GGT (U/L) (range up to 38) & $35.26 \pm 10.03$ & $53.84 \pm 20.30$ & $<0.001$ \\
\hline Total bilirubin (mg/dL) & $0.85 \pm 0.47$ & $1.46 \pm 0.70$ & $<0.001$ \\
\hline Albumin (g/dL) (range 3.2-4.8) & $3.81 \pm 0.33$ & $3.49 \pm 0.52$ & 0.0001 \\
\hline Cholesterol (mg/dL) (range up to 200) & $163.05 \pm 30.28$ & $167.97 \pm 30.64$ & 0.3330 \\
\hline Triglycerides (mg/dL) (range 0-150) & $156.18 \pm 30.31$ & $196.24 \pm 38.07$ & $<0.001$ \\
\hline Fasting blood sugar (mg/dL) (range 60-110) & $115.46 \pm 31.24$ & $134.99 \pm 29.92$ & 0.0002 \\
\hline Fasting insulin $(\mu \mathrm{IU} / \mathrm{mL})$ (range 3-28) & $6.55 \pm 4.15$ & $8.97 \pm 4.30$ & 0.0007 \\
\hline HOMA-IR & $2.06 \pm 1.71$ & $3.13 \pm 1.93$ & 0.001 \\
\hline Chemerin $(\mathrm{ng} / \mathrm{mL})$ & $153.19 \pm 42.72$ & $205.69 \pm 53.22$ & $<0.001$ \\
\hline Adiponectin $(\mu \mathrm{g} / \mathrm{mL})$ & $10.63 \pm 3.13$ & $3.01 \pm 2.07$ & $<0.001$ \\
\hline
\end{tabular}

Data are expressed as mean \pm SD or number (\%)

BMI, body mass index; AST, aspartate aminotransferase; ALT, alanine aminotransferase; GGT, $\gamma$-glutamyl transferase; HOMA-IR, homeostatic model assessmentinsulin resistance; NAFLD, nonalcoholic fatty liver disease

inflammation, ballooning or fibrosis grades. Adiponectin differed significantly within different fibrosis groups $(\mathrm{P}<0.001)$ with its highest mean value in patients with F2, as shown in Table 2 .

\section{Correlation between candidate adipokines and other study parameters}

The correlations of circulating chemerin and adiponectin with other study parameters are shown in Table 3. Chemerin was significantly positively correlated with BMI $(r=0.43$, $\mathrm{P}<0.001)$, AST $(r=0.33, \mathrm{P}=0.001)$, ALT $(r=0.33, \mathrm{P}<0.001)$, GGT $(r=0.35, \mathrm{P}<0.001)$, and triglycerides $(r=0.21, \mathrm{P}=0.01)$, and significantly negatively correlated with adiponectin $(r=-0.36$, $\mathrm{P} \leq 0.001)$. Adiponectin had significant negative correlations with BMI $(r=-0.75, \mathrm{P}<0.001)$, AST $(r=-0.43, \mathrm{P}<0.001)$, ALT $(r=-$ $0.46, \mathrm{P}<0.001)$, GGT $(r=-0.42, \mathrm{P}<0.001)$, alkaline phosphatase $(r=-0.23, \mathrm{P}=0.004)$, triglycerides $(r=-0.58, \mathrm{P}<0.001)$, HOMAIR $(r=-0.27, \mathrm{P}<0.001)$, and chemerin $(r=-0.36, \mathrm{P}<0.001)$.

\section{Predictors of NAFLD diagnosis}

To identify parameters associated with a NAFLD diagnosis, univariate binary logistic regression analyses were performed, with "0" representing the control group and " 1 " representing the NAFLD group. Increased serum AST (OR 1.10, 95\%CI 1.07-1.14, P<0.001), ALT (OR 1.07, 95\%CI 1.05-1.09, $\mathrm{P}<0.001$ ), alkaline phosphatase (OR 1.01, 95\%CI 1.00-1.02, P<0.001), GGT (OR 1.07, 95\%CI 1.04-1.09, $\mathrm{P}<0.001)$, triglycerides (OR 1.04, 95\%CI 1.02-1.05, $\mathrm{P}<0.001$ ), HOMA-IR (OR 1.42, 95\%CI 1.14-1.76, $\mathrm{P}<0.001$ ), and chemerin (OR 1.03, 95\%CI 1.02-1.04, $\mathrm{P}<0.001)$ had a significant positive association with a NAFLD diagnosis. In contrast, increased serum adiponectin was significantly inversely associated with a NAFLD diagnosis (OR 0.52, 95\%CI $0.44-0.62, \mathrm{P}<0.001)$. After adjustment for patients' baseline characteristics in the multivariate adjusted logistic regression model, only GGT (OR 1.07, 95\%CI 1.01-1.13, $\mathrm{P}=0.03$ ), triglycerides (OR 1.96, 95\%CI 0.92-1.00, $\mathrm{P}=0.04$ ), chemerin (OR 1.02, 95\%CI 1.00-1.05, P=0.04) and adiponectin (OR 0.31, 95\%CI 0.16-0.60, $\mathrm{P}<0.001$ ) remained independent predictors for NAFLD, as shown in Table 4.

The best threshold values for the studied adipokines for differentiating NAFLD patients from healthy subjects according to ROC curve analysis are provided in Table 5. ROC analysis demonstrated that a cutoff value of $186.7 \mathrm{ng} / \mathrm{mL}$ for chemerin yielded a sensitivity and specificity of $56.44 \%$ and $87.72 \%$, respectively (C statistics 0.78 ), while a cutoff value of $2.4 \mu \mathrm{g} / \mathrm{mL}$ for adiponectin yielded a sensitivity and specificity of $74.26 \%$ and $3.51 \%$, respectively ( $\mathrm{C}$ statistic $=0.92, \mathrm{P}<0.001$; Fig. 2 ). 
Table 2 Chemerin and adiponectin levels across groups with specific histologic lesions within nonalcoholic fatty liver disease patients $(\mathrm{n}=101)$

\begin{tabular}{|c|c|c|c|}
\hline $\begin{array}{l}\text { Histological } \\
\text { lesion }\end{array}$ & $\begin{array}{c}\text { Patients } \\
\text { N (\%) }\end{array}$ & Chemerin & Adiponectin \\
\hline $\begin{array}{l}\text { Steatosis grade } \\
(\text { P-value })^{*}\end{array}$ & & 0.99 & 0.83 \\
\hline$\leq 33 \%$ & $74(73.27)$ & $205.68 \pm 55.94$ & $3.04 \pm 2.10$ \\
\hline$>33 \%$ & $27(26.73)$ & $205.72 \pm 45.94$ & $2.94 \pm 2.02$ \\
\hline $\begin{array}{l}\text { Lobular } \\
\text { inflammation }{ }^{* *} \\
\text { (P-value) }\end{array}$ & & 0.38 & 0.21 \\
\hline $\begin{array}{l}\leq 4 \text { foci per } \\
\text { (HPF) }\end{array}$ & $49(48.51)$ & $210.52 \pm 53.71$ & $3.28 \pm 2.49$ \\
\hline $\begin{array}{l}>4 \text { foci per } \\
\text { (HPF) }\end{array}$ & $52(51.49)$ & $201.14 \pm 52.87$ & $2.76 \pm 1.56$ \\
\hline $\begin{array}{l}\text { Ballooning } \\
\text { (P-value) }\end{array}$ & & 0.17 & 0.28 \\
\hline Absent & 75 (74.26) & $209.95 \pm 54.10$ & $3.14 \pm 2.34$ \\
\hline Present & $26(25.74)$ & $193.4 \pm 49.57$ & $2.63 \pm 0.84$ \\
\hline $\begin{array}{l}\text { Fibrosis stage } \\
(\text { P-value })^{*}\end{array}$ & & 0.69 & 0.01 \\
\hline F1 & $80(79.21)$ & $208.02 \pm 53.65$ & $2.77 \pm 1.36$ \\
\hline F2 & $14(13.86)$ & $195.93 \pm 54.40$ & $4.58 \pm 4.28$ \\
\hline F3 & $7(6.93)$ & $153.19 \pm 42.72$ & $2.66 \pm 0.74$ \\
\hline
\end{tabular}

Data are expressed as mean $\pm \mathrm{SD}$. ${ }^{*}$ between group comparisons (independent t-test)

HOMA-IR, homeostatic model assessment-insulin resistance; HPF, high power field

Table 3 Correlation between chemerin and adiponectin and other study parameters

\begin{tabular}{lcc}
\hline Parameter & Chemerin & Adiponectin \\
\hline Age $(\mathrm{yrs})$ & $0.00(0.96)$ & $-0.12(0.12)$ \\
BMI $\left(\mathrm{kg} / \mathrm{m}^{2}\right)$ & $0.43(<0.001)$ & $-0.75(<0.001)$ \\
AST & $0.33(<0.001)$ & $-0.43(<0.001)$ \\
ALT & $0.33(<0.001)$ & $-0.46(<0.001)$ \\
GGT & $0.35(<0.001)$ & $-0.42(<0.001)$ \\
Alkaline phosphatase & $0.12(0.14)$ & $-0.23(0.004)$ \\
Triglycerides & $0.21(0.01)$ & $-0.58(<0.001)$ \\
Cholesterol & $0.05(0.50)$ & $0.05(0.53)$ \\
HOMA-IR & $0.15(0.06)$ & $-0.27(<0.001)$ \\
Chemerin & 1 & $-0.36(<0.001)$ \\
Adiponectin & $-0.36(<0.001)$ & 1 \\
\hline
\end{tabular}

Data presented as spearman coefficient of correlation (P-value)

$B M I$, body mass index; AST, aspartate aminotransferase; $A L T$, alanine aminotransferase; GGT, $\gamma$-glutamyl transferase; HOMA-IR, homeostatic model assessment of insulin resistance

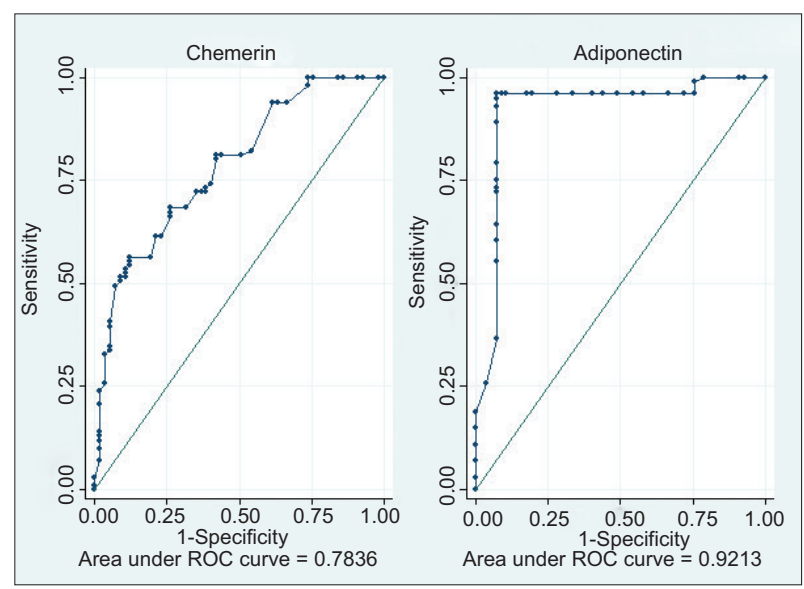

Figure 2 Receiver operator characteristic curves for the diagnostic power of chemerin and adiponectin at various cutoff points in differentiating between patients with nonalcoholic fatty liver disease and controls

\section{Discussion}

In the current case-control study, significantly higher levels of circulating chemerin and significantly lower levels of adiponectin were detected in obese children with biopsy-proven NAFLD compared to controls. Additionally, BMI, AST, ALT, triglycerides, and GGT showed significant positive correlations with chemerin and significant negative correlations with adiponectin.

NAFLD in children is becoming a major health concern, because of its high prevalence and its well-known serious complications, including liver failure and HCC. From 10-30\% of NAFLD patients can progress to liver cirrhosis [14] and $70-90 \%$ of patients with chronic liver disease related to NAFLD develop HCC [15]. Insulin resistance appears to be the key underlying pathophysiological defect leading to NAFLD [16], with subsequent hyperinsulinemia that leads to changes in hepatocyte uptake, synthesis, degradation, and secretion of free fatty acids, and subsequently to the accumulation of triglycerides in the hepatocytes resulting in hepatic steatosis [17-19]. In the present study, we found that HOMA-IR as a surrogate marker for insulin resistance was significantly higher in NAFLD patients compared to controls ( $3.13 \pm 1.93$ vs. $2.06 \pm 1.71$ ). Consistent results have been reported by Kumar et al [20], as well as by Boga et al [21].

In the current investigation, we found that circulating adiponectin levels were significantly lower in NAFLD patients compared to controls $(3.01 \pm 2.07$ vs. $10.63 \pm 3.13 \mu \mathrm{g} / \mathrm{mL}$ respectively). This finding is in keeping with previous studies [2226]. Hypoadiponectinemia might serve as a predictor of NAFLD in obese children [27]. Adiponectin is an abundant adipocyte-derived hormone with well-established antiinflammatory and insulin-sensitizing properties [28]. There is a well-known link between hypoadiponectinemia and NASH, regardless of insulin resistance [29]. Furthermore, within the NAFLD group, a significantly higher level of adiponectin was observed in patients with F2 $(4.58 \pm 4.28 \mu \mathrm{g} / \mathrm{mL})$. This could 
Table 4 Predictors of nonalcoholic fatty liver disease among our cohort by univariate and multivariate logistic regression analyses

\begin{tabular}{|c|c|c|c|c|}
\hline \multirow[t]{2}{*}{ Variables } & \multicolumn{2}{|c|}{ Univariate analysis } & \multicolumn{2}{|c|}{ Adjusted multivariate analysis } \\
\hline & OR $(95 \% \mathrm{CI})$ & P-value & OR (95\%CI) & P-value \\
\hline Age (years) & $1.10(0.98-1.22)$ & 0.09 & $0.89(0.62-1.28)$ & 0.53 \\
\hline Male sex & $0.75(0.37-1.49)$ & 0.41 & $0.61(0.06-6.14)$ & 0.67 \\
\hline $\operatorname{AST}(\mathrm{U} / \mathrm{L})$ & $1.10(1.07-1.14)$ & $<0.001$ & $1.04(0.95-1.13)$ & 0.38 \\
\hline $\operatorname{ALT}(\mathrm{U} / \mathrm{L})$ & $1.07(1.05-1.09)$ & $<0.001$ & $1.02(0.98-1.05)$ & 0.37 \\
\hline Alkaline phosphatase (U/L) & $1.01(1.00-1.02)$ & $<0.001$ & $1.03(0.99-1.07)$ & 0.16 \\
\hline GGT (U/L) & $1.07(1.04-1.09)$ & $<0.001$ & $1.07(1.01-1.13)$ & 0.03 \\
\hline Cholesterol (mg/dL) & $1.01(0.99-1.02)$ & 0.33 & $1.06(1.01-1.13)$ & 0.3 \\
\hline Triglycerides (mg/dL) & $1.04(1.02-1.05)$ & $<0.001$ & $1.96(0.92-1.00)$ & 0.04 \\
\hline HOMA-IR & $1.42(1.14-1.76)$ & $<0.001$ & $0.96(0.48-1.93)$ & 0.91 \\
\hline Chemerin $(\mathrm{ng} / \mathrm{mL})$ & $1.03(1.02-1.04)$ & $<0.001$ & $1.02(1.00-1.05)$ & 0.04 \\
\hline Adiponectin $(\mu \mathrm{g} / \mathrm{mL})$ & $0.52(0.44-0.62)$ & $<0.001$ & $0.31(0.16-0.60)$ & $<0.001$ \\
\hline
\end{tabular}

AST, aspartate aminotransferase; ALT, alanine aminotransferase; GGT, $\gamma$-glutamyl transferase; HOMA-IR, homeostatic model assessment of insulin resistance; OR, odds ratio; 95\%CI, 95\% confidence interval

Table 5 Diagnostic performance, optimal cutoffs, and validity of adiponectin for the diagnosis of nonalcoholic fatty liver disease in a pediatric population

\begin{tabular}{|c|c|c|c|c|c|c|c|}
\hline Variable & Optimal cutoff & P-value & Sensitivity & Specificity & Accuracy & PPV & NPV \\
\hline Chemerin & 186.7 & $<0.001$ & $56.44 \%$ & $87.72 \%$ & 0.7836 & 88.89 & 52.6 \\
\hline Adiponectin & 2.4 & $<0.001$ & $74.26 \%$ & $3.51 \%$ & 0.9213 & 54.7 & 9.7 \\
\hline
\end{tabular}

PPV, positive predictive value; $N P V$, negative predictive value

be attributed to a possible imbalance between adiponectin production and hepatic excretion [30,31], following the increased adiponectin production from the hepatocytes or hepatic stellate cells $[32,33]$.

The most interesting finding is the significantly higher serum chemerin concentration in NAFLD obese children compared to controls $(205.69 \pm 53.22$ vs. $153.19 \pm 42.72 \mathrm{ng} / \mathrm{mL})$. To our best knowledge, such data for the pediatric population are scarce. Our results are in concordance with those reported by Kłusek-Oksiuta et al, who found that chemerin might be a good indicator of liver steatosis in obese children. Chemerin is an adipokine highly expressed in both the liver and adipose tissue [34]; its circulating levels are increased in human obesity [35-37]. It enhances insulin-stimulated glucose uptake and increases insulin sensitivity in adipose tissue [38]. The actual role of chemerin in NAFLD is still unknown.

In conclusion, onclusio circulating chemerin and adiponectin could serve as simple, reliable, noninvasive diagnostic markers for NAFLD in non-diabetic obese children.

\section{Acknowledgment}

The authors wish to thank Ms. Sherif Okasha (Translator at United Nations) who assisted in the proof-reading of the manuscript.

\section{Summary Box}

What is already known:

- Nonalcoholic fatty liver disease (NAFLD) is the most common cause of chronic liver disease in children

- Early diagnosis and proper management of NAFLD could significantly reduce its liver-related morbidity and mortality

- Precise diagnosis of NAFLD is currently dependent on liver histopathology

\section{What the new findings are:}

- Circulating adipokines could serve as noninvasive predictors of NAFLD diagnosis

- Increased circulating chemerin and decreased adiponectin were significantly associated with NAFLD diagnosis in obese non-diabetic children

- Circulating adiponectin differed significantly within different hepatic fibrosis grades 


\section{References}

1. Yki-Järvinen $\mathrm{H}$. Non-alcoholic fatty liver disease as a cause and a consequence of metabolic syndrome. Lancet Diabetes Endocrinol 2014;2:901-910.

2. Bhala N, Jouness RI, Bugianesi E. Epidemiology and natural history of patients with NAFLD. Curr Pharm Des 2013;19:5169-5176.

3. Güngör NK. Overweight and obesity in children and adolescents. J Clin Res Pediatr Endocrinol 2014;6:129-143.

4. Clemente MG, Mandato C, Poeta M, Vajro P. Pediatric non-alcoholic fatty liver disease: Recent solutions, unresolved issues, and future research directions. World J Gastroenterol 2016;22:8078-8093.

5. Anderson EL, Howe LD, Jones HE, Higgins JP, Lawlor DA, Fraser A. The prevalence of non-alcoholic fatty liver disease in children and adolescents: a systematic review and meta-analysis. PLoS One 2015; 10:e140908.5.

6. Almeda-Valdes $\mathrm{P}$, Aguilar-Olivos $\mathrm{N}$, Uribe $\mathrm{M}$, Méndez-Sánchez N. Common features of the metabolic syndrome and nonalcoholic fatty liver disease. Rev Recent Clin Trials 2014;9:148-158.

7. Machado MV, Cortez-Pinto H. Diet, microbiota, obesity, and NAFLD: a dangerous quartet. Int J Mol Sci 2016;17:481.

8. Farrell GC, Larter CZ. Nonalcoholic fatty liver disease: from steatosis to cirrhosis. Hepatology 2006;43:S99-S112.

9. Jamali R. Non-alcoholic fatty liver disease: diagnosis and evaluation of disease severity. Thrita 2013;2:43-51

10. Sumida Y, Nakajima A, Itoh Y. Limitations of liver biopsy and noninvasive diagnostic tests for the diagnosis of nonalcoholic fatty liver disease/nonalcoholic steatohepatitis. World J Gastroenterol 2014;20:475-485.

11. Nobili V, Manco M. Therapeutic strategies for pediatric nonalcoholic fatty liver disease: a challenge for health care providers. World J Gastroenterol 2007;13:2639-2641.

12. Kleiner DE, Brunt EM, Van Natta $M$, et al; Nonalcoholic Steatohepatitis Clinical Research Network. Design and validation of a histological scoring system for nonalcoholic fatty liver disease. Hepatology 2005;41:1313-1321.

13. Yokoyama H, Emoto M, Fujiwara S, et al. Quantitative insulin sensitivity check index and the reciprocal index of homeostasis model assessment in normal range weight and moderately obese type 2 diabetic patients. Diabetes Care 2003;26:2426-2432.

14. Dyson JK, Anstee QM, McPherson S. Non-alcoholic fatty liver disease: a practical approach to treatment. Available from: http:// fg.bmj.com/content/5/4/277. Last accessed: April 2017.

15. Patient Info. Primary Liver Cancer. Available from: http://patient. info/doctor/primary-liver-cancer-pro. Last accessed: April 2017.

16. Cohen DE. Pathogenesis and management of non-alcoholic fatty liver disease. Presented at: National Lipid Association Scientific Sessions; May 19-22, 2016; New Orleans.

17. Kahn BB, Flier JS. Obesity and insulin resistance. J Clin Invest 2000;106:473-481.

18. Houmard JA. Intramuscular lipid oxidation and obesity. Am J Physiol Regul Integr Comp Physiol 2008;294:R1111-R1116.

19. Joseph AM, Joanisse DR, Baillot RG, Hood DA. Mitochondrial dysregulation in the pathogenesis of diabetes: potential for mitochondrial biogenesis-mediated interventions. Exp Diabetes Res 2012;2012:642038.

20. Kumar R, Prakash S, Chhabra S, et al. Association of proinflammatory cytokines, adipokines \& oxidative stress with insulin resistance \& non-alcoholic fatty liver disease. Indian J Med Res 2012;136:229-236.
21. Boga S, Alkim H, Koksal AR, et al. Increased plasma levels of asymmetric dimethylarginine in nonalcoholic fatty liver disease: relation with insulin resistance, inflammation, and liver histology. J Investig Med 2015;63:871-877.

22. Gaddipati R, Sasikala M, Padaki N, et al. Visceral adipose tissue visfatin in nonalcoholic fatty liver disease. Ann Hepatol 2010;9:266-270.

23. Manco M, Marcellini M, Giannone G, Nobili V. Correlation of serum TNF-alpha levels and histologic liver injury scores in pediatric nonalcoholic fatty liver disease. Am J Clin Pathol 2007;127:954-960.

24. Wieckowska A, Papouchado BG, Li Z, Lopez R, Zein NN, Feldstein AE. Increased hepatic and circulating interleukin-6 levels in human nonalcoholic steatohepatitis. Am J Gastroenterol 2008; 103:1372-1379.

25. Chu CJ, Lu RH, Wang SS, et al. Plasma levels of interleukin-6 and interleukin-8 in Chinese patients with non-alcoholic fatty liver disease. Hepatogastroenterology 2007;54:2045-2048.

26. Senates E, Yilmaz Y, Colak Y, et al. Serum levels of hepcidin in patients with biopsy-proven nonalcoholic fatty liver disease. Metab Syndr Relat Disord 2011;9:287-290.

27. Zou CC, Liang L, Hong F, Fu JF, Zhao ZY. Serum adiponectin, resistin levels and non-alcoholic fatty liver disease in obese children. Endocr J 2005;52:519-524.

28. Finelli C, Tarantino G. What is the role of adiponectin in obesity related non-alcoholic fatty liver disease? World J Gastroenterol 2013;19:802-812.

29. Lemoine M, Ratziu V, Kim M, et al. Serum adipokine levels predictive of liver injury in non-alcoholic fatty liver disease. Liver Int 2009;29:1431-1438.

30. Tietge UJ, Böker KH, Manns MP, Bahr MJ. Elevated circulating adiponectin levels in liver cirrhosis are associated with reduced liver function and altered hepatic hemodynamics. Am J Physiol Endocrinol Metab 2004;287:E82-E89.

31. Hui CK, Zhang HY, Lee NP, et al; Hong Kong Liver Fibrosis Study Group. Serum adiponectin is increased in advancing liver fibrosis and declines with reduction in fibrosis in chronic hepatitis $\mathrm{B}$. J Hepatol 2007;47:191-202.

32. Brunt EM. Nonalcoholic steatohepatitis: definition and pathology. Semin Liver Dis 2001;21:3-16.

33. Ding X, Saxena NK, Lin S, et al. The roles of leptin and adiponectin: a novel paradigm in adipocytokine regulation of liver fibrosis and stellate cell biology. Am J Pathol 2005;166:1655-1669.

34. Kłusek-Oksiuta M, Bialokoz-Kalinowska I, Tarasów E, Wojtkowska M, Werpachowska I, Lebensztejn DM. Chemerin as a novel non-invasive serum marker of intrahepatic lipid content in obese children. Ital J Pediatr 2014;40:84.

35. Bozaoglu K, Bolton K, McMillan J, et al. Chemerin is a novel adipokine associated with obesity and metabolic syndrome. Endocrinology 2007;148:4687-4694.

36. Parlee SD, Ernst MC, Muruganandan S, Sinal CJ, Goralski KB. Serum chemerin levels vary with time of day and are modified by obesity and tumor necrosis factor-\{alpha\}. Endocrinology 2010;151:2590-2602.

37. Stejskal D, Karpisek M, Hanulova Z, Svestak M. Chemerin is an independent marker of the metabolic syndrome in a Caucasian population-a pilot study. Biomed Pap Med Fac Univ Palacky Olomouc Czech Repub 2008;152:217-221.

38. Takahashi M, Takahashi Y, Takahashi K, et al. Chemerin enhances insulin signaling and potentiates insulin-stimulated glucose uptake in 3T3-L1 adipocytes. FEBS Lett 2008;582:573-578. 